\title{
An alinement chart for the evaluation of the dynamic modulus of elasticity and Poisson's ratio for concrete*
}

by W. H. King, B.A., B.Sc., A.M.I.C.E.,

and I. D. G. Lee, B.Sc.(Eng.)

A.M.(S.A.)I.C.E.

\section{Contribution by J. C. Simmons, M.Sc.(Eng.)}

The note by King and Lee is of considerable interest since the writer has recently published a paper (1) in which results from a large number of non-destructive tests are summarized. These tests included measurements of longitudinal resonant frequency and ultrasonic pulse velocity, and both the dynamic elastic modulus and Poisson's ratio were determined. These determinations could evidently have been made from a nomogram such as is now suggested.

Before referring to the nomogram, however, consideration might be given to the basic equations given on page 165 , since there appear to be grounds for confusion concerning the units.

For longitudinal resonance in the fundamental mode, and using the notation of King and Lee, the fundamental physical equation is

$$
2 n L=\sqrt{\frac{E_{1}}{\rho}} \ldots \ldots \ldots \ldots \ldots \ldots
$$

The units in this equation should, of course, be consistent and thus it is most convenient to use the usual physical units, viz.

$$
\begin{aligned}
n & =\text { frequency }(\text { cycles } / \mathrm{sec}) \\
L & =\text { length }(\mathrm{ft}) \\
E_{1} & \left.=\text { elastic modulus (poundals } / \mathrm{ft}^{2}\right) \\
\rho & =\text { density }\left(\mathrm{lb} / \mathrm{ft}^{3}\right)
\end{aligned}
$$

Here the unit of force is taken as the poundal and that of mass as the pound. The use by King and Lee of $l b / \mathbf{f t}^{2}$ for $E_{1}$ and $l b$ (mass) $/ \mathrm{ft}^{3}$ for $\rho$ does not appear to be consistent with the usual definitions of these units, unless by $l b$ (mass) is meant slug. This latter unit is, however, rarely used in this type of engineering work, and it is probably better avoided.

Considering the rewritten form of equation (1) in terms of more commonly used units, viz.

$$
2 n l=\sqrt{\frac{12^{4} g E}{w}}
$$

*Pages 165-168. the quoted units are:

$$
\begin{aligned}
l & =\text { length (in.) } \\
E & =\text { elastic modulus }\left(\mathrm{lb} / \mathrm{in}^{2}\right) \\
g & =\text { gravitational acceleration }\left(\mathrm{ft} / \mathrm{sec}^{2}\right) \\
w & =\text { density }\left(\mathrm{lb}\left[\text { weight] } / \mathrm{ft}^{3}\right)\right.
\end{aligned}
$$

$E$ is clearly now in $l b($ weight $) / \mathrm{in}^{2}$. Density, however, is not weight $/ \mathrm{ft}^{3}$ as suggested by King and Lee, but can only be mass $/ \mathrm{ft}^{3}$, and therefore there seems to be no reason why the original symbol, $p$, should not be used again, where $p=$ density in $l b / \mathrm{ft}^{3}$ and the pound is again the unit of mass.

The units which should be used in equation (2) would thus seem to be:

$$
\begin{array}{ll}
n \text { cycles } / \mathrm{sec} \\
l \text { in. } \\
E \mathrm{lb} / \mathrm{in}^{2} \\
g \mathrm{ft} / \mathrm{sec}^{2} \\
w=\rho \mathrm{lb} / \mathrm{ft}^{3}
\end{array}
$$

It is noticed that King and Lee do not refer to the lateral inertia correction to the longitudinal resonant frequency, though, within the limits covered by their chart, this correction may become appreciable. As the authors say, under resonant conditions lateral stresses in the bar are absent. Conversely, however, lateral strains are present, and these strains can cause significant effects on the resonant behaviour, dependent on the properties and dimensions of the test specimen. This subject has been considered in some detail elsewhere ${ }^{(2,3)}$, but briefly, the kinetic energy of the accompanying lateral vibration causes a reduction of resonant frequency below that which would obtain if the specimen were such that lateral vibrations were negligible.

The value of $n$ to be used in equation (1) or (2) is the true resonant frequency in the absence of lateral vibrations and, subject to certain simplifying assumptions, this can be shown to be related to the observed resonant frequency, $n^{\prime}$, by the relation

$$
n=n^{\prime}\left(1+\frac{m^{2} \pi^{2}}{L^{2}} \sigma^{2} \frac{J}{2 A}\right)
$$


where $m=$ an integer depending on the mode of vibration;

$\sigma=$ Poisson's ratio for the material of the bar;

$J=$ polar second moment of cross-sectional area of bar;

$A=$ cross-sectional area of bar.

For bars of square cross-section vibrating in the fundamental mode this reduces to

$$
n=n^{\prime}\left(1+\frac{\pi^{2}}{12} \sigma^{2} \frac{D^{2}}{L^{2}}\right)
$$

where $D=$ length of side of cross-section.

The appropriate value of $\sigma$ to be used in this equation is open to doubt, since it has been shown ${ }^{(1)}$ that more than one value of Poisson's ratio may be determinable for the same concrete specimen. The correction to the observed resonant frequency is, however, generally fairly small, and the value of $\sigma$ taken is probably not critical.

This correction is mentioned here since for specimens, say, $16 \times 4 \times 4$ in. at an age of 1 day with $\sigma \bumpeq 0 \cdot 30$, the correction to $n^{\prime}$ would amount to about $\frac{1}{2} \%$. This value could be significant when compared with the sensitivity quoted for the chart. For most purposes, however, the correction can generally be ignored.

Referring now to the proposed nomogram, the authors suggest that tedious calculations are involved in the determination of $\sigma$ from measurements of dynamic elastic modulus and ultrasonic pulse velocity. This, however, need not be so. The theoretical relationship for pulse velocity, in the same units as suggested above for equation (1), is

$$
V=\sqrt{\frac{E_{1}}{\rho} \frac{(1-\sigma)}{(1+\sigma)(1-2 \sigma)}} \cdots \cdots \cdots
$$

where $V$ is given in $\mathrm{ft} / \mathrm{sec}$.

Equations (1) and (4) thus give

$$
\frac{n L}{V}=\sqrt{\frac{(1+\sigma)(1-2 \sigma)}{4(1-\sigma)}}
$$

But if $t$ (sec) is the time taken for the pulse to traverse the length $L$ of the specimen, then

$$
V=\frac{L}{t}
$$

whence

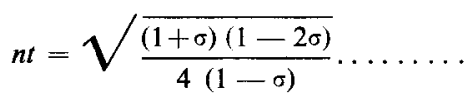

Thus Poisson's ratio, $\sigma$, may be plotted directly in terms of the product $n t$ as in Figure A. Now both $n$ and $t$ are quantities directly measured in the appropriate tests. Thus $\sigma$ may be determined quite simply from the product of these two quantities which are actually measured.

It is true that often the value of $V$ is required for other reasons and thus it has to be calculated anyway, but

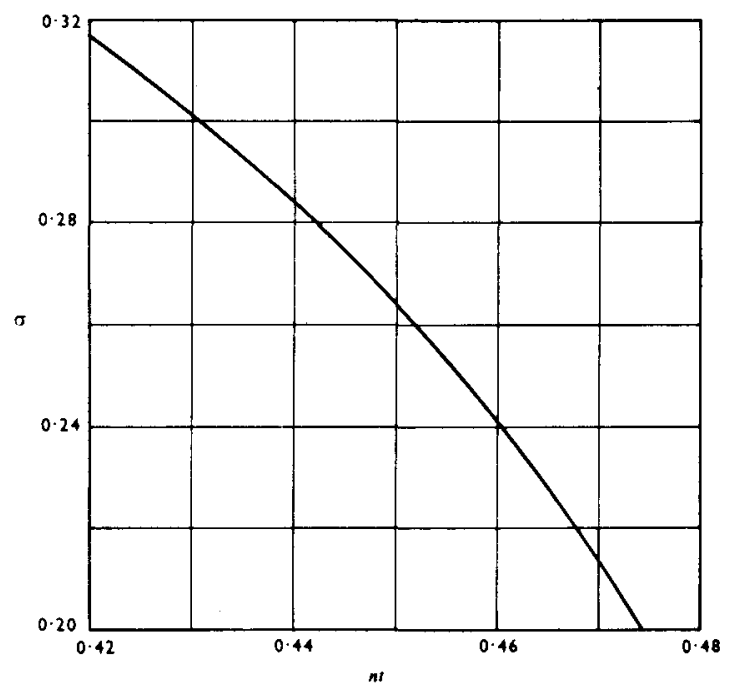

Figure A: Chart for determining Poisson's ratio from longitudinal resonant frequency and ultrasonic pulse transmission time.

allowing for this it does not seem that the method proposed above is any more arduous than the method using the nomogram.

Rewriting equation (2) in the form

$$
E=\frac{4}{12^{4} g} w n^{2} l^{2}
$$

it can be seen that to evaluate $E$ using a slide rule or a desk computor would take little, if any, longer than the equivalent steps using the nomogram.

The nomogram proposed by King and Lee is quite large and thus is possibly a little difficult to store flat without folding. Furthermore, in spite of the suggestion of a length of clear plastic with a scribed straight line, the nomogram will probably often be marked up with pencil, and it is well known that this soon leads to damage. The nomogram is, however, readily available in a prepared form and thus the rather trying task of its preparation no longer confronts the would-be user.

In conclusion, my experience suggests that if large numbers of calculations are to be made at any one time, then the use of the nomogram might be preferred, but that if the calculations are to be done on a day to day basis immediately following comparatively small numbers of tests, then a simple graph and slide rule method as suggested above may be preferable.

\section{REFERENCES}

11) SIMMONs, J. C. Poisson's ratio of concrete: a comparison of dynamic and static measurements. Magazine of Concrete Research. Vol. 7, No. 20. July 1955. pp. 61-68. 
(2) CHREE, C. On longitudinal vibrations. The Quarterly Journal of Pure and Applied Mathematics. Vol. 23. 1889. pp. 317-342.

(3) SIMmons, J. C. An investigation of Poisson's ratio for concrete. Thesis presented to the University of London for the degree of M.Sc. 1954. pp. 128-136.

\section{Reply by I. D. G. Lee}

In Mr King's absence, I have been asked to reply to the points raised by $\mathrm{Mr}$ Simmons.

The confusion over units is unfortunate since the authors attempted to define each stage in the transformation of the equations. In designing the alinement chart it was desired to observe the notation used in Clause 105 of B.S. 1881. To link this notation with that of the basic physical equations, it was assumed that $p=w / g$ as anticipated by $\mathrm{Mr}$ Simmons in introducing the term "slug ". By this device, gravitational units of force (pound weight) may be used throughout. If, however, absolute units of force (poundal) are used in the basic equation, then the notation used by $\mathrm{Mr}$ Simmons is equally correct.

The correction of the observed resonant frequency for lateral inertia might well be accomplished by adding an extra scale to the alinement chart, $(D / L)^{2}$. The example taken for a $16 \times 4 \times 4$ in. specimen at an age of 1 day with $\sigma=0.30$ is rather an extreme case, since Poisson's ratio is known to decrease rapidly with age. The test outlined in Clause 105 of B.S. 1881 requires the measurement of frequency to an accuracy of $\pm \frac{1}{2} \%$, the shortest specimen having a length of $20 \mathrm{in.}$ Within these limits the lateral inertia is insensible. On page 167 it is stated that the sensitivities of some of the scales were greater than could be observed at that time, anticipating improvements in the apparatus. If these improvements now justify a correction for lateral inertia, a separate alinement chart for this purpose is preferable to further complicating the existing chart.

The final point raised by Mr Simmons is a matter of taste. Either you like nomograms or you do not. A graph for determining $\sigma$ similar to that submitted by $\mathrm{Mr}$ Simmons was originally used at the Research Division of the Cement and Concrete Association. As the volume of routine non-destructive testing increased it became necessary to provide a chart which could be understood and used by operators and laboratory assistants who might not be expert users of a slide-rule. The chart had been successfully used for one year prior to its publication in the Magazine of Concrete Research. 